\title{
Mudanças no perfil da mulher vítima de violência sexual em uma capital do sul do brasil
}

Gabriela Calixto Maluf ${ }^{1}$ (D), Isabela Casari Donida ${ }^{1}$ (D), Jean A. Furtado Correa Francisco ${ }^{2}$ (D), Renato Nisihara ${ }^{1}$

\section{RESUMO}

Introdução: O perfil da mulher vítima de violência sexual deve ser conhecido para elaboração de medidas preventivas.

Objetivos: Avaliar o perfil epidemiológico e a aplicação do protocolo de atendimento para mulheres vítimas de violência sexual nos casos acolhidos no Hospital Universitário Evangélico Mackenzie (HUEM), em Curitiba-PR.

Metodologia: Foram avaliados os dados da Ficha de Violência Interpessoal/Autoprovocada de mulheres com idade maior ou igual a 12 anos, acolhidas no serviço de atenção à vítima de violência sexual do HUEM em Curitiba-PR, entre janeiro de 2015 a dezembro de 2018. Foram buscados dados sociodemográficos da vítima, da ocorrência em si, clínicos e laboratoriais, sobre o provável autor da violência e sobre o retorno ao serviço.

Principais resultados: Foram estudadas 252 vítimas de violência sexual. No período, a idade das vítimas nos anos 2015 e 2016 (mediana de 19 anos) em relação aos anos 2017 e 2018 (mediana de 17 anos) reduziu de forma significativa $(p=0,026)$. Já a escolaridade não influenciou a violência sexual $(p=0,64)$ e ser solteira conferiu maior risco $(p<0,0001 ; O R=16,1)$. O horário noturno foi o de maior risco $(p<0,0001 ; O R=3,5)$. Dentre as vítimas com idade entre 12 e 18 anos, 63,6\% dos agressores são conhecidos e nas maiores de 18 anos, 70,8\% dos agressores são desconhecidos $(p<0,0001)$. Observou-se que $46 \%$ das vítimas não retornaram para nenhuma das consultas previstas e apenas $1,6 \%$ cumpriram o protocolo de acompanhamento.

Conclusão: No período estudado, a redução significativa da idade apontou uma mudança no perfil demográfico da mulher vítima de violência sexual. Mesmo em um serviço de referência, a taxa de retorno ainda é muito baixa e são necessárias medidas para aumentar a adesão ao protocolo.

Palavras-chave: Violência sexual, Mulheres, Epidemiologia

\section{INTRODUÇÃO}

A violência sexual é definida como uma ação não consensual de conjunção carnal ou outro ato libidinoso que transgride a autonomia da vítima (1). Essa forma de violência gera profundo impacto na vida das vítimas nas esferas física, psíquica e social, além de ter relação direta com prejuízos à saúde mental $(2,3)$.

No Brasil, o cenário predominante da subnotificação se mantém $(4,5)$, com uma taxa de notificação policial de apenas 7,5\% (6). Apesar disso, dados do Anuário de Segurança Pública mostram que o número de casos de estupro está aumentando. Revelou-se um crescimento nos casos de $10,1 \%$ de 2016 para 2017 e de $4,1 \%$ de 2017 para 2018, chegando ao cenário atual de 180 estupros por dia $(7,8)$. Junto a esse aumento, o quadro nacional de violência sexual aponta que $81,8 \%$ dos casos são do sexo feminino, sendo $53,8 \%$ com menos de 13 anos e $50,9 \%$ negras. Além disso, mulheres solteiras com baixa escola-

${ }_{1}^{1}$ Faculdade Evangélica Mackenzie do Paraná, (PR), Brasil

${ }^{2}$ Hospital Universitário Evangélico Mackenzie, Curitiba, (PR), Brasil 
ridade apresentaram maior vulnerabilidade com relação a essa tipologia criminal (9).

Políticas públicas norteadas pela legislação e aplicadas no Sistema Único de Saúde são desenvolvidas como parte de um esforço do poder público para reduzir os danos e a frequência da violência sexual (10). O atendimento obrigatório e integral às vítimas de violência sexual é garantido pela Lei n.o 12.845, sancionada em 2013. Um dos centros de referência para o atendimento dessas vítimas em Curitiba é o Hospital Universitário Evangélico Mackenzie (HUEM), onde são atendidas vítimas de violência sexual (VVS) do sexo feminino e com mais de 12 anos. Esse atendimento inicial busca acolher, prevenir gestação, infecções sexualmente transmissíveis, oferecer atendimento psicológico e acompanhamento para a vítima, previsto por até seis meses após a data de agressão para o controle periódico laboratorial, além de possíveis tratamentos e acompanhamento gestacional (11).

O conhecimento do perfil epidemiológico das vítimas, dos agressores e do contexto em que ocorreu a violência sexual é de extrema importância para a elaboração de medidas preventivas e melhor entendimento dos casos. Além disso, o acompanhamento e a eficácia de intervenções para prevenir doenças e a gravidez devem ser analisados com periodicidade para atualização e mudanças nos pontos falhos do protocolo.

Os objetivos desse trabalho foram determinar o perfil epidemiológico das vítimas, da ocorrência e dos agressores nos casos de violência sexual acolhidos no HUEM no período de janeiro de 2015 a dezembro de 2018, avaliar as intervenções feitas no atendimento inicial das vítimas e analisar a taxa de retorno das pacientes ao serviço de referência.

\section{MÉTODOS}

Esse estudo tem desenho retrospectivo e observacional a partir de coleta de dados. Foi aprovado pelo Comitê de Ética em Pesquisa da Sociedade Evangélica Beneficente de Curitiba-PR com o número CAAE 02788418.1.0000.0103.

Foram incluídas no estudo todas as mulheres com idade maior ou igual a 12 anos admitidas no serviço de atenção à vítima em situação de violência sexual no HUEM entre janeiro de 2015 e dezembro de 2018. Foram excluídas fichas de pacientes que não tinham pelo menos $75 \%$ dos dados requisitados para o estudo. A vítima não foi identificada por nome ou iniciais, apenas pelo código de atendimento.

Os dados que constavam da Ficha de Violência Interpessoal/ Autoprovocada (12), e do sistema informatizado do ambulatório de ginecologia do HUEM coletados foram os seguintes:

Dados da vítima: número de atendimento, data da notificação, hora do atendimento, município e estado da notificação, unidade notificadora, data da ocorrência da violência, data de nascimento, idade, sexo, gestante, raça/cor, escolaridade, dados da residência, situação conjugal/ estado civil, ocupação, orientação sexual, identidade de gênero, deficiência/transtorno.

Dados da ocorrência em si: município e estado da ocorrência, distrito, bairro, logradouro, número e complemento, ponto de referência, zona, hora da ocorrência, local da ocorrência, ocorrência de outras vezes, lesão autoprovocada, motivação da violência, tipo da violência, meio de agressão, natureza da agressão, tipo de violência sexual, violência relacionada ao trabalho.

Dados clínicos e laboratoriais: natureza da lesão, parte do corpo atingida, procedimento realizado.

Dados do provável autor da violência: número de envolvidos, vínculo com a pessoa atendida, sexo do agressor, suspeita de álcool e de outras drogas, faixa etária do provável autor da violência.

Dados sobre retorno: dados de encaminhamento e número de retorno ambulatorial.

\section{Análise estatística}

Os dados coletados foram planilhados com auxílio do programa Excel $\circledast$. As análises estatísticas serão feitas com o auxílio do programa GraphPad Prism 6.0. As variáveis contínuas foram expressas como média \pm desvio-padrão ou medianas e interquartis, sendo comparadas com os testes t e Mann-Whitney. As variáveis categóricas foram expressas em porcentagens e comparadas com o teste do qui-quadrado ou teste exato de 
Fisher, conforme apropriado. Valores de $\mathrm{p}$ menores que $5 \%$ foram considerados estatisticamente significativos.

\section{RESULTADOS}

No período entre janeiro de 2015 e dezembro de 2018 foram atendidas no HUEM 264 muIheres VVS. Pelos critérios estabelecidos, foram excluídos 12 casos. Dessa forma, foram investigadas no presente estudo 252 VVS.

A Tabela 1 apresenta características sociodemográficos das pacientes analisadas. A mediana de idade no período foi de 18 anos (IIQ= 14 - 25 anos), com idade mínima de 12 anos e a máxima de 68 anos. A mediana de idade foi diminuindo com a evolução dos anos, partindo de 19 anos em 2015 até 17 anos em 2018, dado observado na Figura 1. Essa redução se mostrou significativa $(p=0,026)$ quando foram comparadas as idades das VVS dos anos 2015 e 2016 [mediana de 19 anos (IIQ = 14 a 29 anos) ] em relação aos anos 2017 e 2018 [mediana de 17 anos (IIQ = 14 a 23 anos)]. Ainda em relação à faixa etária, torna-se importante ressaltar que $47,6 \%(120 / 252)$ das vítimas tinham idade menor que 18 anos. A escolaridade não influenciou a violência sexual $(p=0,64)$ e o estado civil solteiro conferiu maior risco $(p<0,0001 ;$ OR $=16,1 ;$ IC95\% $=10,2-25,5)$. Adicionalmente, $13,5 \%$ das VVS eram portadoras de algum tipo de deficiência ou transtorno.

Em relação aos dados da ocorrência da violência sexual, disponibilizados na Tabela 1, observou-se que $90,8 \%$ dos casos ocorreram em zona urbana e $37,6 \%$ em ambiente residencial. Ressalta-se que $61,9 \%$ dos casos ocorreram durante a noite $(n=156)$, conferindo o horário da noite como de maior risco ( $p<0,0001 ; \mathrm{OR}=3,5$; IC95\% $=2,4-5,1)$. Histórico de violência sexual anterior ao atendimento foram relatadas por $17,8 \%$ das vítimas $(n=45)$.

Os dados relacionados à violência sexual podem ser analisados na Tabela 2. Estupro foi a violência mais frequente $(61,9 \%)$, tanto no aspecto físico como emocional. Observou-se que $52,8 \%(133 / 252)$ dos agressores eram desconhecidos, enquanto $43,7 \%$ (110/252) tinham algum vínculo com as vítimas. Dentre as vítimas com idade entre 12 e 18 anos, 63,6\% (84/120) relataram que os agressores eram conhecidos; já no grupo das vítimas com mais de 18 anos, em $70,8 \%(85 / 120)$ dos casos os agressores foram relatados como desconhecidos $(p<0,0001)$. Espancamento foi relatado em $47,6 \%$ dos casos e na maioria dos casos $(66,7 \%)$ a violência foi cometida por um único agressor. Em 36,5\% dos casos as VVS relatam que o agressor estava sob efeito do uso de álcool.

A Figura 2a disponibiliza os dados referentes às intervenções realizadas no atendimento inicial das vítimas acolhidas no serviço. Nota-se que $42,1 \%$ das VVS realizaram coleta de sêmen e que $80 \%$ realizaram coleta de sangue para realização de exames laboratoriais. Profilaxia para doenças infecciosas foi realizada em cerca de $70 \%$ dos casos. Em relação ao seguimento das pacientes, na Figura $\mathbf{2 b}$ pode ser observado que $46 \%(116 / 252)$ das vítimas não retornaram para nenhuma das consultas previstas pelo Protocolo Para o Atendimento às Pessoas em Situação de Violência Sexual e que apenas $1,6 \%$ realizaram quatro ou mais consultas de retorno, ou seja, concluíram as orientações do protocolo.

\section{DISCUSSÃO}

Em 2018, 53.726 mulheres foram vítimas de violência sexual no Brasil (9). Isso significa que a cada hora cerca de seis mulheres foram vítimas de estupro. Diante disso, torna-se fundamental conhecer o perfil das vítimas e das ocorrências para que se apliquem as devidas medidas de prevenção e de acolhimento. Nosso estudo contribui com dados que ajudam a desenhar esse perfil na região sul, além de evidenciar uma mudança na idade das vítimas, cada vez mais jovens, que deve ser analisada ativamente em outras regiões do país a fim de avaliar se há um novo padrão de vítimas e de agressores que requer novas formas de atuação do poder público.

A predominância de vítimas menores de idade é compatível com a literatura em relação à maior prevalência de vítimas jovens, principalmente com menos de 20 anos (13). Outro dado importante levantado pelo estudo foi a significativa diminuição da mediana de idade de 19 anos 
em 2015 para 17 anos em 2018. Isso evidencia uma mudança no perfil epidemiológico das VVS que deve ser explorada para balizar as medidas preventivas de segurança pública e para se adequar os serviços de referência responsáveis pelo acolhimento.

Cerca de $60 \%$ das vítimas se identificou como sendo da raça branca, dado semelhante ao encontrado em São Paulo, mas não compatível com o encontrado em Recife $(14,15)$. A discordância entre os estudos possivelmente se deve à diversidade regional que caracteriza o nosso país. Outro ponto relevante observado no presente estudo foi que a escolaridade não constituiu fator de risco para violência sexual, enquanto a literatura retrata que mulheres com baixa escolaridade são mais vulneráveis $(14,15)$. É possível que tal achado seja devido ao maior acesso à escolarização que ocorreu nos últimos anos em várias regiões do Brasil. Outra possibilidade é que as mulheres com maior escolaridade tenham perdido o receio de denunciar os abusos ocorridos.

O estado civil solteiro é amplamente relatado na literatura como fator de risco para a violência sexual (14-18). Nesse estudo, mulheres solteiras tiveram até 25 vezes mais risco de se tornarem vítimas. Esse achado deve ser usado para prevenção da violência sexual, aumentando a informação para esse grupo. Por outro lado, acreditamos que tal dado pode ser resultante de um viés, pois se acredita que as mulheres solteiras VVS são as que mais buscam atendimento médico e legal.

A maioria dos casos ocorreu no período da noite, fator de risco significativo para a ocorrência da violência sexual citado na literatura $(14,15,17,18)$. Adicionalmente, esse estudo apontou que via pública e ambiente residencial foram os locais com maior frequência de ocorrências, dado compatível com estudo realizado em Teresina (17), mas não observado por outros autores $(14,15,18)$.

A partir de 2009 (lei 12.015), houve uma mudança do Artigo 2013 do Código Penal que definiu como estupro: "Constranger alguém, mediante violência ou grave ameaça, a ter conjunção carnal ou a praticar ou permitir que com ele se pratique outro ato libidinoso". Anteriormente à modificação, considerava-se crime de estupro apenas se este fosse constituído de conjunção carnal. Acreditamos que essa mudança permite uma visão mais verossímil da violência sexual como um espectro formado pelas diferentes formas de agressão à vítima, o que contribuiria tanto para melhor acolhimento, quanto para garantir amparo legal em casos de violência sexual sem conjunção carnal. Com a mudança da lei e, consequentemente, dos protocolos de atendimento, esse espectro deve ser conhecido pelo profissional de saúde responsável pelo atendimento inicial à VVS. O tipo de violência sexual mais referido pelas vítimas foi o estupro, seguido do assédio sexual, dado compatível com a literatura (15). Em metade das agressões houve violência física e também violência psicológica. A variedade de formas pelas quais as vítimas sofreram violência reforçam a necessidade de medidas que assegurem o acolhimento e acompanhamento, visto que as consequências afetam diretamente a qualidade de vida dessas mulheres (2).

Em relação aos agressores, aproximadamente $25 \%$ das ocorrências tiveram dois ou mais agressores. Tal número foi maior que o observado por outros autores $(14,17,18)$. Adicionalmente, um terço das vítimas relata uso de álcool pelo agressor, sendo similar ao observado em Teresina (PI) (17).

Metade das vítimas relata que sofreu a violência sexual por desconhecidos. Porém, quando dividimos a amostra em dois grupos etários, vítimas de até 18 anos e com mais de 18 anos, o primeiro grupo relatou maioria de agressores conhecidos e, o segundo, agressores desconhecidos, dado similar ao observado na literatura (18). Tal achado aponta que as intervenções para prevenção de violência sexual devem levar em consideração as diferentes faixas etárias. São necessárias políticas públicas de educação sexual em escolas para conscientizar as mulheres mais jovens e encorajá-las a denunciar os agressores, conhecidos ou não. Por outro lado, para vítimas adultas e idosas, tal política não se aplicaria, uma vez que os agressores são em sua maioria desconhecidos. Nesse grupo, a prevenção mais efetiva seria a melhoria da segurança pública. O ponto de convergência para ambos os grupos é a necessidade de apoio e proteção após a ocorrência. Independentemente da idade, o acolhimento das vítimas 
é fundamental, seja no âmbito legal (delegacias), seja em ambientes médicos como centros de referência de atendimento às vítimas. O atendimento, solidário, humanizado e de qualidade pode prevenir infecções sexualmente transmissíveis, gravidez indesejada e principalmente tentar minimizar as consequências psicológicas que afetam diretamente a vida dessas mulheres (2).

Em relação aos procedimentos realizados no primeiro atendimento, mais de $70 \%$ das pacientes coletou material para exames e recebeu a profilaxia preconizada para tal evento, valores similares aos descritos por outros autores (11-14). Ressalta-se a importância dessa conduta para prevenção de maiores agravos. No entanto, em relação aos retornos para a consulta ambulatorial de acompanhamento prevista pelo Protocolo Para o Atendimento às Pessoas em Situação de Violência Sexual, 40\% das mulheres compareceram a uma só consulta e apenas $1,6 \%$ das vítimas completaram o seguimento ambulatorial. Tais taxas são um pouco superiores das observadas em outro serviço de referência de Curitiba (19), mas ainda muito inferiores às recomendadas. A baixa taxa de retorno já foi referida por outros autores (20). No nosso entendimento, os profissionais de saúde que acolhem as VVS no serviço avaliado estão preparados para tal função. No entanto, consideramos necessário aprimorar a assistência às VVS e realizar educação continuada dos profissionais envolvidos no atendimento. (2). Além disso, cabe ressaltar que o seguimento ambulatorial sofre forte influência negativa de aspectos emocionais da vítima. É conhecido que as VVS se sentem constrangidas em retornar ao ambulatório, pois retornar é relembrar a experiência traumatizante que gostariam de esquecer. É possível que esse seja o principal fator responsável pela baixa taxa de adesão ao protocolo de acompanhamento estabelecido.

Esse estudo apresenta algumas limitações devido ao seu desenho retrospectivo. Alguns dados das fichas de notificação são marcados como ignorados ou estão incompletos. No entanto, acredita-se que os dados principais foram repassados. Também se ressalta que uma grande parcela de mulheres, ao sofrerem a violência sexual não procuram os serviços de saúde, por vários motivos.

\section{CONCLUSÃO}

Em nosso estudo, o perfil da vítima de violência sexual mostrou predominância de muIheres brancas, solteiras e jovens, sendo que a mediana de idade diminuiu ao longo dos anos estudados. A ocorrência mais frequente foi estupro, no período da noite. O agressor foi predominantemente conhecido em vítimas menores de 18 anos e desconhecido em vítimas maiores de 18 anos. Após o acolhimento, a maioria das vítimas recebeu profilaxia para ISTs e contracepção de emergência. Apesar do acompanhamento médico e psicológico estar disponível, $46 \%$ das vítimas não retornou para nenhuma das consultas ambulatoriais previstas.

\section{REFERÊNCIAS}

1. Valle R, Bernabé-Ortiz A, Gálvez-Buccollini J, Gutiérrez C, Martins S. Intrafamilial and extrafamilial sexual assault and its association with alcohol consumption. Rev Saude Publica. 2018;52.

2. Labronici LM, Fegadoli D, Correa MEC. Significado da violência sexual na manifestação da corporeidade: um estudo fenomenológico. Rev da Esc Enferm. 2010;44(2):401-6.

3. Tarzia L, Thuraisingam S, Novy K, Valpied J, Quake R, Hegarty $K$. Exploring the relationships between sexual violence, mental health and perpetrator identity: A cross-sectional Australian primary care study. BMC Public Health. $2018 ; 18(1) 1410$.

4. Conceição M, Costa O, Santos CAST. O perfil da violência contra crianças e adolescentes, segundo registros de Conselhos Tutelares: vítimas, agressores e manifestações de violência. Ciência \& Saúde Coletiva, 12(5):1129-1141. https://dx.doi.org/10.1590/ S1413-81232007000500010

5. Souza C de M e, Adesse L. Violência sexual no Brasil: perspectivas e desafios. 2005 186p. Available from: http:// bases.bireme.br/cgi-bin/wxislind.exe/iah/online/?IsisScrip$\mathrm{t}=\mathrm{iah} / \mathrm{iah} \cdot \mathrm{xis} \& \mathrm{src}=$ google\&base $=$ ADOLEC\&lang $=\mathrm{p} \&$ nextAc tion $=$ Ink\&exprSearch $=429888 \&$ indexSearch $=$ ID

6. Nacional P. Pesquisa Nacional de Vitimização Questionário SENASP Maio de 2013 Pesquisa Nacional de Vitimização Questionário SENASP. 2013; Available from: http://www. crisp.ufmg.br/wp-content/uploads/2013/10/Relat $\square$ rio-PNV-Senasp_final.pdf

7. Anuário Brasileiro de Segurança Pública [Internet]. 2017 [cited 2020 Sep 26]. Available from: www.forumseguranca.org.br

8. Anuário Brasileiro de Segurança Pública 2018. Fórum Bras Segurança Pública [Internet]. 2018;1-90. Available 
from: http://www.forumseguranca.org.br/wp-content/ uploads/2019/03/Anuario-Brasileiro-de-Segurança-Pública-2018.pdf

9. Anuário Brasileiro de Segurança Pública 2019. https://www.forumseguranca.org.br/wp-content/uploads/2019/10/Anuario-2019-FINAL_21.10.19.pdf

10. Pinto LSS, de Oliveira IMP, Pinto ESS, Leite CBC, Melo A do $N$, de Deus MCBR. Políticas públicas de proteção à mulher: Avaliação do atendimento em saúde de vítimas de violência sexual. Ciencia e Saude Coletiva. 2017;22(5):1501-8.

11. Andrade, Rosires Pereira de. Violência sexual contra muIheres: aspectos médico, psicológicos, sociais e legais do atendimento. 2. ed. Curitiba: Faculdade de Medicina da Universidade Federal do Paraná, 2017.

12. Brasil. Ministério da Saúde. Ficha de Notificação de Violência Interpessoal/Autoprovocada [cited 2020 Sep 26]. Available from: https://portalarquivos2.saude.gov.br/images/pdf/2015/julho/02/Ficha-Viol-5.1-Final_15.06.15.pdf

13. Andrade RP, Guimarães ACP, Fagotti Filho Á, Carvalho NS de, Arrabal JS, Rocha DM da, et al. Características Demográficas e Intervalo para Atendimento em Mulheres Vítimas de Violência Sexual. Rev Bras Ginecol e Obs. 2001;23(9):583-7.

14. Facuri $C$ de $O$, Fernandes AM dos $S$, Oliveira KD, Andrade TDS, de Azevedo RCS. Violência sexual: Estudo descritivo sobre as vítimas e o atendimento em um service universitário de referência no estado de São Paulo, Brasil. Cad Saude Publica. 2013;29(5):889-98.
15. Nunes MCA, Lima RFF, Morais NA de. Violência Sexual contra Mulheres: um Estudo Comparativo entre Vítimas Adolescentes e Adultas. Psicol Ciência e Profissão. 2017;37(4):956-69.

16. Sousa TCC de, Coelho ASF, Mattos DV de, Valadares JG, Lima MRG de, Costa PS, et al. Características de mulheres vítimas de violência sexual e abandono de seguimento de tratamento ambulatorial. Cad Saúde Coletiva. 2019; 27(2):117-23. Available from: https://www.scielo.br/j/ cadsc/a/Hvz3wtBKQFRr3CgDhHZypXq/?lang=pt

17. Araujo RP de, Sousa FM da S, Feitosa VC, Coêlho DMM, Sousa M de FA e. Perfil sociodemográfico e epidemiológico da violência sexual contra as mulheres em Teresina/ Piauí. Rev Enferm da UFSM. 2015;4(4):739-50.

18. Cerqueira D, Coelho D. Estupro no Brasil: uma radiografia segundo os dados da Saúde (versão preliminar). IPEANota Técnica no 11 [Internet]. 2014;1-30. Available from: http://www.ipea.gov.br/portal/index.php?option=com_content\&view=article\&id=21842

19. Trigueiro TH, Merighi MAB, Medeiros ARP de, Ribeiro CEL, Mata NDS da, Jesus MCP de. Vítimas De Violência Sexual Atendidas Em Um Serviço De Referência. Cogitare Enferm. $2015 ; 20(2): 249-56$

20. Neto JA, Colas OR, Bianca G, Bertolani M. Assistência multiprofissional à vítima de violência sexual : a experiência da Universidade Federal de São Paulo Multidisciplinary care for victims of sexual assault : the experience at the Federal University in São Paulo, Brazil. Cad. Saúde Pública. 2007;23(2):459-64. 
TABELA 1 - CARACTERÍSTICAS SOCIODEMOGRÁFICAS DAS MULHERES VÍTIMAS DE VIOLÊNCIA SEXUAL E DADOS DA OCORRÊNCIA DOS CASOS ATENDIDOS NO HUEM ENTRE 2015 E 2018

Variável

Idade (anos)

$12-15$

$18-29$

30-59

Acima de 60

\section{Gestante}

Sim

Não/ignorado

\section{Raça/cor}

Branca

Preta

Outras

\section{Escolaridade}

Analfabetos

Ensino Fundamental

Ensino Médio

Ensino Superior

Ignorado

Identidade de gênero

Mulher transexual

Ignorado

Estado Civil

Solteiro

Não solteiro

\section{Deficiência/Transtorno}

Sim

Não/Ignorado

Tipo de Deficiência/ Transtorno

Transtorno mental 13

Intelectual

Auditiva

Transtorno de comportamento

Outros
250

15

237

151

8

93

3

112

92

29

16

185

67

34

218

13

12

6

6

5
0,8

$\%$

47,6

32,8

18,9

0,4

6

93,9

59,9

3,2

36,9

1,2

44,4

36,5

11,5

6,3

99,2

73,4

26,5

13,5

86,5

5,1

4,8

2,4

2,4

2 
Visual

Física

Zona

Urbana

Rural

Outro

Horário

Manhã (6-12h59)

Tarde (13-17h59)

Noite (18-5h59)

Ignorado

Local de ocorrência

Residência

Via pública

Escola

Outro

Antecedente pessoal de violência sexual

Sim

Não

Ignorado
2

1

229

7

16

44

39

156

13

95

93

2

62

45

182

25
0,8

0,4

90,8

2,7

6,3

17,4

15,4

61,9

5,1

37,6

37

0,8

24,3

17,8

72,2

10 
TABELA 2 - DADOS DA VIOLÊNCIA E DO AGRESSOR DOS CASOS ANTENDIDOS NO HUEM ENTRE 2015 E 2018

Variável

n

Tipo de violência sexual

Estupro

156

Assédio sexual

Exploração sexual

Pornografia infantil

80

16

4

\section{Violência}

Física

113

Psicológica

85

Associada a negligência ou abandono

16

Tortura

Financeira

Relacionada ao tráfico de mulheres

\section{5}

4

2

\section{Natureza da lesão}

Espancamento

Ameaça

64

Outros

Enforcamento

Objeto perfurocortante

Arma de fogo

Envenenamento

Objeto contundente

Substância quente

\section{Dados do agressor}

Um agressor

66,7

Dois ou mais agressores

Desconhecido

62

133

110

92

61,9

31,7

6,3

1,6

44,8

33,7

6,3

2

1,6

0,8

47,6

25,4

15,1

3,6

3,2

2,4

1,6

0,4

0,4

Conhecido

Uso de álcool pelo agressor

24,6

52,8

43,7

36,5 
TABELA 3 - DADOS DA VIOLÊNCIA E DO AGRESSOR DOS CASOS ANTENDIDOS NO HUEM ENTRE 2015 E 2018

\begin{tabular}{|c|c|c|}
\hline Variável & $\mathbf{n}$ & $\%$ \\
\hline \multicolumn{3}{|l|}{ Tipo de violência sexual } \\
\hline Estupro & 156 & 61,9 \\
\hline Assédio sexual & 80 & 31,7 \\
\hline Exploração sexual & 16 & 6,3 \\
\hline Pornografia infantil & 4 & 1,6 \\
\hline \multicolumn{3}{|l|}{ Violência } \\
\hline Física & 113 & 44,8 \\
\hline Psicológica & 85 & 33,7 \\
\hline $\begin{array}{l}\text { Associada a negligência ou aban- } \\
\text { dono }\end{array}$ & 16 & 6,3 \\
\hline Tortura & 5 & 2 \\
\hline Financeira & 4 & 1,6 \\
\hline $\begin{array}{l}\text { Relacionada ao tráfico de mulhe- } \\
\text { res }\end{array}$ & 2 & 0,8 \\
\hline \multicolumn{3}{|l|}{ Natureza da lesão } \\
\hline Espancamento & 120 & 47,6 \\
\hline Ameaça & 64 & 25,4 \\
\hline Outros & 38 & 15,1 \\
\hline Enforcamento & 9 & 3,6 \\
\hline Objeto perfurocortante & 8 & 3,2 \\
\hline Arma de fogo & 6 & 2,4 \\
\hline Envenenamento & 4 & 1,6 \\
\hline Objeto contundente & 1 & 0,4 \\
\hline Substância quente & 1 & 0,4 \\
\hline \multicolumn{3}{|l|}{ Dados do agressor } \\
\hline Um agressor & 168 & 66,7 \\
\hline Dois ou mais agressores & 62 & 24,6 \\
\hline Desconhecido & 133 & 52,8 \\
\hline Conhecido & 110 & 43,7 \\
\hline Uso de álcool pelo agressor & 92 & 36,5 \\
\hline
\end{tabular}

Fonte: Os autores (2019). 
Figura 1 - Intervenções realizadas no atendimento inicial às vítimas de violência sexual atendidas no Hospital Universitário Evangélico Mackenzie entre 2015 e 2018 ( $n=252)$

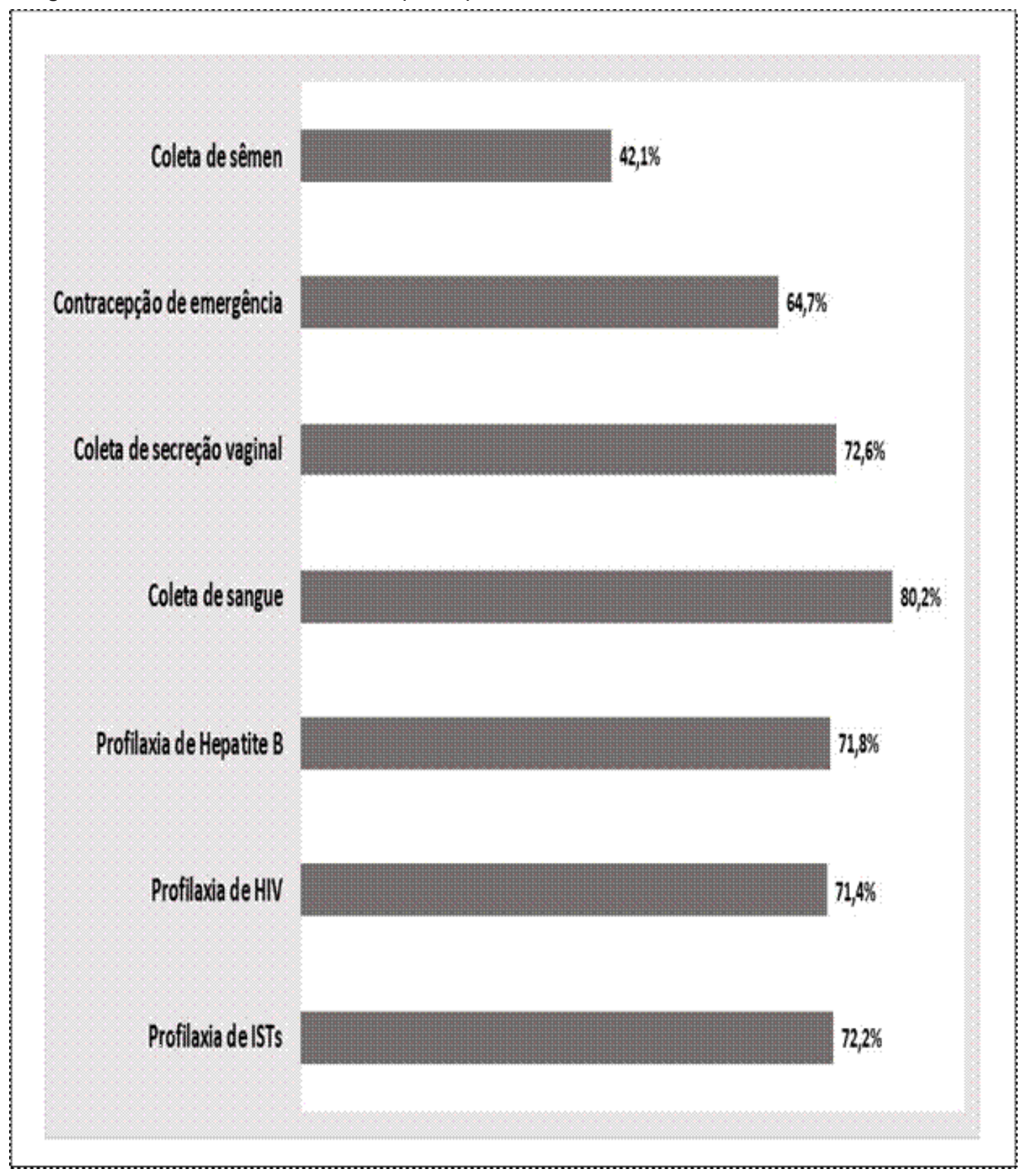


Figura 2. Número de retornos ao ambulatório das vítimas de violência sexual atendidas no Hospital Universitário Evangélico Mackenzie entre 2015 e 2018 (n=252)

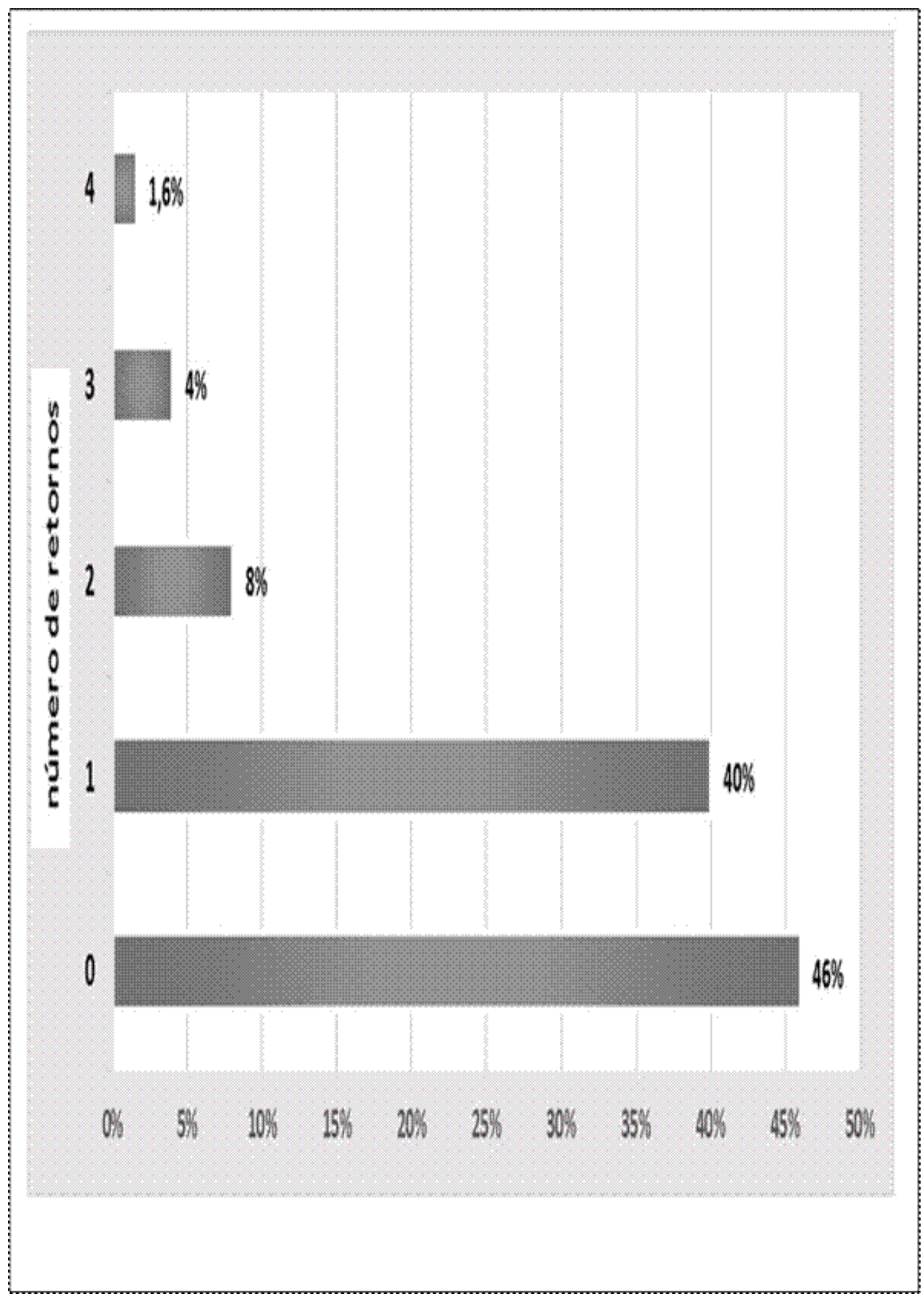




\section{Conflitos de Interesses:}

Os autores declaram não ter conflitos de interesse.

Apoio financeiro:

O estudo não recebeu nenhum apoio financeiro.

Autor Correspondente:

Prof. Renato Nisihara

renatonisihara@gmail.com;

renato.nisihara@fempar.edu.br

Editor:

Prof. Dr. Marcelo Riberto

Recebido em: 03/11/2020

Aprovado em: 09/02/2021

cC) (i) Este é um artigo publicado em acesso aberto (Open Access) sob a licença Creative

sem restrições, desde que o trabalho original seja corretamente citado. 